\title{
PIECEWISE MONOTONE INTERPOLATION AND APPROXIMATION WITH MUNTZ POLYNOMIALS
}

BY

ELI PASSOW, LOUIS RAYMON AND OVED SHISHA

ABSTRACT. The possibility (subject to certain restrictions) of solving the following approximation and interpolation problem with a given set of "Muntz polynomials" on a real interval is demonstrated:

(i) approximation of a continuous function by a "copositive" Muntz polynomial;

(ii) approximation of a continuous function by a "comonotone" Muntz polynomial;

(iii) approximation of a continuous function with a monotone $k$ th difference by a Muntz polynomial with a monotone $k$ th derivative;

(iv) interpolation by piecewise monotone Muntz polynomials-i.e., polynomials that are monotone on each of the intervals determined by the points of interpolation.

The strong interrelationship of these problems is shown implicitly in the proofs.

The following related questions have been settled:

I (Monotone Approximation). Let $f(x)$ be a continuous function with the property that the $j$ th difference $\Delta^{j} f \geqslant 0$ on $[0,1]$ where $j$ is some nonnegative integer. Must there be for a given $\epsilon>0$ a corresponding polynomial $p(x)$ with $p^{(j)}(x) \geqslant 0$ on $[0,1]$ such that

$$
\|f-p\|=\sup _{x \in[0,1]}|f(x)-p(x)|<\epsilon ?
$$

II (Comonotone Approximation). Let $f(x)$ be a continuous function with a finite number of nodes on $[0,1]$; i. e., suppose $0=x_{0}<x_{1}<\cdots<x_{k}=1$ and that $f(x)$ is alternately nondecreasing and nonincreasing on the intervals $\left(0, x_{1}\right),\left(x_{1}, x_{2}\right), \ldots,\left(x_{k-1}, x_{k}\right)$. For a given $\epsilon>0$ must there be a corresponding polynomial $p(x)$ that has the same monotonicity as $f(x)$ on each of the intervals $\left(x_{i-1}, x_{i}\right), i=1,2, \ldots, k$, and such that $\|f-p\|<\epsilon$ ?

Received by the editors October 31, 1973.

AMS (MOS) subject classifications (1970). Primary 41A05, 41A10, 41 A30, 41 A25.

Key words and phrases. Approximation, interpolation, Muntz polynomials, restricted approximation, restricted interpolation, monotone approximation, piecewise monotone interpolation.

The authors wish to acknowledge D. Myers for pointing out errors in the manuscript. 
III (Piecewise Monotone Interpolation). Let $X=\left(x_{0}, x_{1}, \ldots, x_{n}\right)$ and $Y=\left(y_{0}, y_{1}, \ldots, y_{n}\right)$ where $0=x_{0}<x_{1}<\cdots<x_{n}=1$ and $y_{i} \neq y_{i-1}$, $i=1,2, \ldots, n$. Is there a polynomial $p$ such that $p\left(x_{i}\right)=y_{i}, i=0,1, \ldots, n$, and such that $p(x)$ is monotone in each of the intervals $\left(x_{i-1}, x_{i}\right), i=1,2, \ldots, n$ ?

The answer to $I$ is in the affirmative, by the properties of the Bernstein polynomials [5]. II and III also have affirmative answers, as established by Wolibner [12], Kammerer [2] and Young [13]. Furthermore, quantitative estimates have been obtained for the degree $n(\epsilon)$ of the polynomial that is sufficient for monotone and comonotone approximation [11], [10], [6], [4], [9], [7], and for the degree $n(X, Y)$ of the polynomial that is sufficient for piecewise monotone interpolation [8]. By the classic Theorem of Muntz, if $0=\lambda_{0}, \lambda_{1}$, $\lambda_{2}, \ldots$ is a sequence of distinct nonnegative real numbers such that $\lambda_{i}+0$, then the "Muntz polynomials" $\Sigma_{i=0}^{N} a_{i} x^{\lambda_{i}}$ are dense in $C[0,1]$ if and only if $\Sigma\left(1 / \lambda_{i}\right)=\infty$. (If the interval $[0,1]$ is replaced by $[a, b]$ with $a>0$, then the restriction $\lambda_{0}=0$ may be omitted.) A natural question to ask is: Are monotone approximation, comonotone approximation and/or piecewise monotone interpolation possible with Muntz polynomials? In this article we answer these three questions in the affirmative (for "admissible" sets $\Lambda=\lambda_{0}, \lambda_{1}, \ldots$ ) and, for the case of monotone approximation we obtain quantitative estimates for this approximation. Theorems 2 and 3, concerning, respectively, comonotone approximation and piecewise monotone interpolation, and Theorem 1 have proofs that are interrelated.

THEOREM 1. Let $[0,1]$ be partitioned into $k$ subintervals by the points $0=x_{0}<x_{1}<\cdots<x_{k}=1$. Suppose that $f(x)$ is a continuous function that is alternately nonnegative and nonpositive on the intervals $\left(0, x_{1}\right),\left(x_{1}, x_{2}\right), \ldots$, $\left(x_{k-1}, 1\right)$. Let $\Lambda=\left\{\lambda_{0}, \lambda_{1}, \ldots\right\}$ be a sequence of nonnegative real numbers with the following properties: (i) $0,1, \ldots, k-1 \in \Delta$; (ii) $\lim \lambda_{i}=\infty$; (iii) $\Sigma\left(1 / \lambda_{i}\right)=\infty$. Then, given $\epsilon>0$ there is a corresponding Muntz polynomial $p(x)=\sum_{i=0}^{N} a_{i} x^{\lambda_{i}}$ such that $p(x)$ is copositive with $f(x)$ (i.e., $p(x)$ is nonnegative on those intervals of the partition in which $f$ is nonnegative and $p(x)$ is nonpositive on those intervals of the partition on which $f$ is nonpositive) and such that $\|f-p\|<\epsilon$.

THEOREM 2. Let $[0,1]$ be partitioned into $k$ subintervals by the points $0=x_{0}<x_{1}<\cdots<x_{k}=1$. Suppose that $f(x)$ is a continuous function that is alternately nondecreasing and nonincreasing on the intervals $\left(0, x_{1}\right),\left(x_{1}, x_{2}\right)$, $\ldots,\left(x_{k-1}, 1\right)$. Let $\Lambda=\left\{\lambda_{0}, \lambda_{1}, \ldots\right\}$ be a sequence of nonnegative real numbers with the following properties: (i) $0,1, \ldots, k \in \Lambda$; (ii) $\lim \lambda_{i}=\infty$; (iii) $\Sigma\left(1 / \lambda_{i}\right)=\infty$. Then, given $\epsilon>0$ there is a corresponding Muntz polynomial $p(x)=\Sigma_{i=0}^{N} a_{i} x^{\lambda_{i}}$ such that $p(x)$ is comonotone with $f(x)$ (i. e., $p(x)$ is nonde- 
creasing on those intervals of the partition on which $f$ is nondecreasing, and $p(x)$ is nonincreasing on those intervals of the partition on which $f$ is nonincreasing) and such that $\|f-p\|<\epsilon$.

Theorem 3. Let $X=\left(x_{0}, x_{1}, \ldots, x_{n}\right)$ and $Y=\left(y_{0}, y_{1}, \ldots, y_{n}\right)$ where $0=x_{0}<x_{1}<\cdots<x_{n}=1$ and $y_{i} \neq y_{i-1}, i=1,2, \ldots, n$. Let $k-1$ be the number of "vertices" $\left(x_{i}, y_{i}\right)$ at which the piecewise linear function joining the points $\left(x_{0}, y_{0}\right),\left(x_{1}, y_{1}\right), \ldots,\left(x_{n}, y_{n}\right)$ changes monotonicity $(k \leqslant n)$. Let $\Lambda=\left\{\lambda_{0}, \lambda_{1}, \ldots\right\}$ be a sequence of nonnegative real numbers with the following properties: (i) $0,1, \ldots, k \in \Lambda$; (ii) $\lim \lambda_{i}=\infty$; (iii) $\Sigma\left(1 / \lambda_{i}\right)=\infty$. Then there is a Muntz polynomial $p(x)=\sum_{i=0}^{N} a_{i} x^{\lambda_{i}}$ such that $p\left(x_{i}\right)=y_{i}, i=0,1, \ldots, n$, and such that $p(x)$ is monotone in each of the intervals $\left(x_{i-1}, x_{i}\right), i=1,2, \ldots, n$.

Denote by $\mathrm{T}_{k}$ Theorem 1 for the case where the number of intervals in the partition is $k, k=1,2, \ldots$; denote by $\mathrm{T} 2{ }_{k}$ Theorem 2 for the case where the number of intervals in the partition is $k$; denote by $\mathrm{T} 3_{k}$ Theorem 3 for the case where $k-1$ is the number of vertices at which the described piecewise linear function changes monotonicity.

Theorems 1, 2 and 3 follow immediately from the following sequence of lemmas.

LEMMA 1. $\mathrm{T1}_{1}$.

LEMMA 2. $\mathrm{T}_{k} \Rightarrow \mathrm{T} 2_{k}, k=1,2, \ldots$.

LEMMA 3. $\mathrm{T} 2_{k} \Rightarrow \mathrm{T} 3_{k}, k=1,2, \ldots$

LEMMA 4. $\mathrm{T} 3_{k} \Rightarrow \mathrm{T} 1_{k+1}, k=1,2$. .

Lemma 1 is trivial: Suppose $f(x) \geqslant 0$ on $[0,1]$ and let $\epsilon>0$ be arbitrary. Then, by Muntz' Theorem, there is a Muntz polynomial $p(x)$ such that $\|f-p\|<$ $\epsilon / 2$. Then $p^{*}(x)=p(x)+\epsilon / 2$ is a Muntz polynomial satisfying

and

$$
p^{*}(x)=\epsilon / 2+p(x)-f(x)+f(x) \geqslant \epsilon / 2+f(x)-\|f-p\|>0,
$$

$$
\left\|f-p^{*}\right\| \leqslant\|f-p\|+\left\|p-p^{*}\right\|<\epsilon
$$

Proof of Lemma 2. Let $f(x)$ and $\Lambda$ be as described in Theorem 2 , and let $\epsilon>0$ be arbitrary. Let $f^{*}(x)$ be defined on $[0,1]$ with the following properties:

(i) $f^{*}$ has the same nodes as $f\left(\right.$ i.e., at $\left.x_{i}, i=1,2, \ldots, k-1\right)$ and is alternately nondecreasing and nonincreasing on the same intervals as $f$;

(ii) $f^{*} \in C^{1}[0,1]$;

(iii) $\left\|f^{*}-f\right\|<\epsilon / 2$.

$\left(f^{*}\right.$ may be chosen to be a spline approximation to $f$ with knots at the nodes, 
with care taken to ensure the monotonicity property.)

Then $g(x)=d f^{*}(x) / d x$ is a continuous function on $[0,1]$ that is alternately nonnegative and nonpositive on the intervals on which $f^{*}$ is alternately nondecreasing and nonincreasing. Furthermore if $\Lambda^{\prime}=\left\{\lambda_{l}^{\prime}=\lambda_{i}-1: \lambda_{i} \in \Lambda\right.$ and $\left.\lambda_{i} \geqslant 1\right\}$, then $\Lambda^{\prime}$ satisfies the requirements (i), (ii) and (iii) of $\mathrm{Tl}_{k}$. Then, by $\mathrm{Tl}_{k}$, there is a Muntz polynomial $q(x)=\Sigma_{i=0}^{N} b_{i} x^{\lambda_{i}}$ that is copositive with $g(x)$ such that $\|g-q\|<\epsilon / 2$. Let $p(x)=\int_{0}^{x} q(x) d x+f^{*}(0)$. Then $p$ is comonotone with $f^{*}$ (hence, with $f$ ) and

$$
\left\|p-f^{*}\right\|=\left\|\int_{0}^{x}[g(x)-q(x)] d x\right\| \leqslant\|g-q\|<\epsilon / 2,
$$

hence

$$
\|p-f\| \leqslant\left\|p-f^{*}\right\|+\left\|f^{*}-f\right\|<\epsilon .
$$

Proof of Lemma 3. Let $X, Y$ and $\Lambda$ be given subject to the conditions in $\mathrm{T}_{k}$, and let $L(x)$ be the piecewise linear function described in the theorem. Then $L(x)$ is alternately increasing and decreasing on $k$ subintervals partitioning $[0,1]$. Let $L=L(X, Y, \epsilon)$ be the set of all possible piecewise linear functions joining $\left(x_{0}, y_{0} \pm \epsilon\right),\left(x_{1}, y_{1} \pm \epsilon\right), \ldots,\left(x_{n}, y_{n} \pm \epsilon\right) . \quad L$ consists of $2^{n+1}$ piecewise linear functions $L_{1}, L_{2}, \ldots, L_{2^{n+1}}$. Choose $\epsilon>0$ so small that $L_{i}(x)$ is comonotone with $L(x)$ for each $1 \leqslant i \leqslant 2^{n+1}$. For each $L_{i}$ there is, by $\mathrm{T} 2_{k}$, a corresponding Muntz polynomial $p_{i}$ comonotone with $L_{i}$ (hence, with $L$ ) such that $\left\|L_{i}-p_{i}\right\|<\epsilon / 2$. Then, $Y$ is in the convex hull (in $E^{n+1}$ ) of the points

$$
\left\{p_{i}(X)\right\}=\left\{\left(p_{i}\left(x_{0}\right), \ldots, p_{i}\left(x_{n}\right)\right)\right\}, \quad 0 \leqslant i \leqslant 2^{n+1} .
$$

Hence $Y=\Sigma_{i=0}^{2^{n+1}} b_{i} p_{i}(X)$, with $b_{i} \geqslant 0$ for all $i$. We observe that linear combinations of Muntz polynomials are Muntz polynomials, and that positive linear combinations of functions comonotone with $L(x)$ are comonotone with $L(x)$. Consequently, $p(x)=\Sigma_{i=1}^{2^{n+1}} b_{i} p_{i}(x)$ is a Muntz polynomial with the desired properties.

Proof of Lemma 4. Let $f(x)$ be a continuous function that is alternately nonnegative and nonpositive on the $k+1$ intervals in a partition of $[0,1]$ determined by the nodes $0=x_{0}<x_{1}<\cdots<x_{k+1}=1$, and let $\Lambda$ be as described in $\mathrm{Tl}_{k+1}$. Suppose, first, that $f$ is not constant on any of the intervals $\left(x_{i-1}, x_{i}\right)$, $i=1,2, \ldots, k+1$. Let

$$
0<\epsilon<\delta=\min _{1<i<k+1} \max _{i-1<x<x_{i}}|f(x)| .
$$

For each point $x_{i}$ of the $k$ points $x_{1}, \ldots, x_{k}$ at which $f(x)$ changes sign let $a_{i}, b_{i}$ be defined as follows:

$$
a_{i}=\max _{x<x_{i}}\{x:|f(x)| \geqslant \epsilon / 6\} ; \quad b_{i}=\min _{x>x_{i}}\{x:|f(x)| \geqslant \epsilon / 6\} .
$$


It follows from (1) that $A=\bigcup\left(a_{i}, b_{i}\right)$ form $k$ nonoverlapping intervals. The complement $\mathrm{C} A$ of $A$ consists of $k+1$ intervals on which $f$ is alternately nonnegative and nonpositive. Let

$$
f^{*}(x)=\left\{\begin{array}{l}
f(x), \quad x \in A, \\
\max [f(x), \epsilon / 6], \text { on intervals of } \mathrm{C} A \text { on which } f \text { is nonnegative, } \\
\min [f(x),-\epsilon / 6], \text { on intervals of } \mathrm{C} A \text { on which } f \text { is nonpositive. }
\end{array}\right.
$$

Observe that

(i) $f^{*}$ is continuous, and copositive with $f$;

(ii) $\left|f^{*}(x)\right| \leqslant \epsilon / 6$ for $x \in A$;

(iii) $\left|f^{*}(x)\right| \geqslant \epsilon / 6$ for $x \in \mathrm{C} A$;

(iv) $f^{*}\left(x_{i}\right)=0, i=1, \ldots, k$;

(v) $\left\|f-f^{*}\right\| \leqslant \epsilon / 6$.

Let $f^{* *}$ be a function with the following properties:

(i) $f^{* *}$ is alternately nonnegative and nonpositive on the intervals $\left(x_{0}, x_{1}\right), \ldots,\left(x_{k}, x_{k+1}\right)$ (i.e., $f^{* *}$ is copositive with $f^{*}$ and $f$ );

(ii) $f^{* *} \in C^{1}[0,1]$;

(iii) at each point $x_{i}$ that $f(x)$ changes from nonnegative to nonpositive, $d f^{* *}\left(x_{i}\right) / d x=-1$; at each point $x_{i}$ that $f(x)$ changes from nonpositive to nonnegative, $d f^{* *}\left(x_{i}\right) / d x=1$;

(iv) $\left\|f^{* *}-f^{*}\right\|<\epsilon / 12$.

$f^{* *}$ will then have the following additional properties:

(v) $\left|f^{* *}(x)\right| \leqslant\left|f^{*}(x)\right|+\left\|f^{* *}-f^{*}\right\|<\epsilon / 4$ for $x \in A$;

(vi) $\left|f^{* *}(x)\right| \geqslant\left|f^{*}(x)\right|-\left\|f^{* *}-f^{*}\right\|>\epsilon / 12$ for $x \in \mathrm{C} A$;

(vii) $\left\|f^{* *}-f\right\| \leqslant\left\|f^{* *}-f^{*}\right\|+\left\|f^{*}-f\right\|<\epsilon / 4$.

Let $g(x)=d f^{* *}(x) / d x$, and let $\Lambda^{\prime}=\left\{\lambda_{i}^{\prime}=\lambda_{i}-1 ; \lambda_{i} \in \Lambda\right.$ and $\left.\lambda_{i} \geqslant 1\right\}$. By Muntz' Theorem there is a Muntz polynomial $q(x)=\sum_{i=0}^{N} c_{i} x^{\lambda_{i}}$ such that $\|g-q\|$ $<\epsilon / 24$. Let $r(x)=\int_{0}^{x} q(x) d x+f^{* *}(0)$. Then $r$ is a Muntz polynomial with respect to $\Lambda$, and

(i) $\left\|r-f^{* *}\right\|=\left\|\int_{0}^{x}[g(x)-q(x)] d x\right\| \leqslant\|g-q\|<\epsilon / 24$.

In particular,

(ii) $\left|r\left(x_{i}\right)\right|<\epsilon / 24, i=1, \ldots, k$.

Also

(iii) $\|r-f\| \leqslant\left\|r-f^{* *}\right\|+\left\|f^{* *}-f\right\|<7 \epsilon / 24$.

(iv) For $x \in A,|r(x)| \leqslant\left|f^{* *}(x)\right|+\left\|r-f^{* *}\right\|<7 \epsilon / 24$.

(v) For $x \in \mathrm{C} A, r$ is copositive with $f$, and

$$
|r(x)| \geqslant\left|f^{* *}(x)\right|-\left\|r-f^{* *}\right\|>\epsilon / 24 .
$$

If $f(x)$ is changing from nonnegative to nonpositive at $x_{i}$, since $r^{\prime}\left(x_{i}\right)$ is close to -1 , there must be an interval $\left(c_{i}, d_{i}\right)$ such that $r(x)$ is decreasing on $\left(c_{i}, d_{i}\right)$. 
Also, if $f$ is changing from nonpositive to nonnegative at $x_{i}, r^{\prime}\left(x_{i}\right)$ is close to 1 , and there must be an interval $\left(c_{i}, d_{i}\right)$ such that $r$ is increasing on $\left(c_{i}, d_{i}\right)$. Clearly, $c_{i}, d_{i}$ may be chosen such that $a_{i}<c_{i}<x_{i}<d_{i}<b_{i}$.

Let $L(x)$ be a piecewise linear function with the following properties:

(i) $L(x)$ alternately decreases and increases $k$ times; $L(x)$ is decreasing on each interval $\left(a_{i}, b_{i}\right)$ containing a point $x_{i}$ at which $f$ changes from nonnegative to nonpositive, and is increasing on each interval $\left(a_{i}, b_{i}\right)$ containing a point $x_{i}$ at which $f$ changes from nonpositive to nonnegative. (None of the segments of $L(x)$ is parallel to the $x$-axis.)

(ii) $L(x)$ has vertices at $x_{i}, c_{i}, d_{i}, i=1, \ldots, k ; L\left(x_{i}\right)=-r\left(x_{i}\right) ;\left|L\left(c_{i}\right)\right| \geqslant$ $7 \epsilon / 24 ;\left|L\left(d_{i}\right)\right| \geqslant 7 \epsilon / 24$.

(iii) $\|L(x)\|<17 \epsilon / 24$.

Applying T $3_{k}$ where $X$ and $Y$ are determined by the vertices of $L(x)$, there is a Muntz polynomial $s(x)$ with the following properties:

(i) $s\left(x_{i}\right)=-r\left(x_{i}\right), i=1, \ldots, k$;

(ii) $s$ is copositive with $f$ on the complement of the intervals $\left(c_{i}, d_{i}\right), i=$ $1, \ldots, k$;

(iii) $s$ is alternately decreasing and increasing on the intervals $\left(a_{i}, b_{i}\right), \ldots$, $\left(a_{k}, b_{k}\right)$;

(iv) $|s(x)| \geqslant 7 \epsilon / 24$ on the intervals $\left(a_{i}, c_{i}\right),\left(d_{i}, b_{i}\right), i=1, \ldots, k$;

(v) $\|s(x)\|<17 \epsilon / 24$.

Let $p(x)=r(x)+s(x)$. Then $p(x)$ is a Muntz polynomial with $p\left(x_{i}\right)=0$, $i=1,2, \ldots, k$. We show that $p$ and $f$ are copositive: since $r$ and $s$ are both copositive with $f$ on $\mathrm{C} A, p$ is copositive with $f$ on this set. For points $x_{i}$ at which $f$ changes from nonnegative to nonpositive, $r$ and $s$ are both nonincreasing on the corresponding intervals $\left(c_{i}, d_{i}\right)$; hence their sum $p$ is nonincreasing on these intervals (and passes through 0 at $x_{i}$ ), and $f$ and $p$ are copositive on the interval $\left(c_{i}, d_{i}\right)$. Similarly, $f$ and $p$ are copositive on intervals $\left(c_{i}, d_{i}\right)$ containing points $x_{i}$ at which $f$ changes from nonpositive to nonnegative. On the remaining points, $\left(a_{i}, c_{i}\right),\left(b_{i}, d_{i}\right), i=1, \ldots, k, f$ and $s$ are copositive. Also, $|s(x)| \geqslant 7 \epsilon / 24 \geqslant$ $|r(x)|$, and $p$ must be copositive with s. Hence $p$ and $f$ are copositive throughout $[0,1]$.

Finally,

$$
\|p-f\| \leqslant\|r-f\|+\|s\|<7 \epsilon / 24+17 \epsilon / 24=\epsilon,
$$

and the proof is complete for the case where $f$ is not constant on any of the intervals $\left(x_{i-1}, x_{i}\right), i=1, \ldots, n$. This case may be dispensed with by taking a continuous copositive approximation $\bar{f}$ of $f$ that is not constant on any of the intervals, and approximating $\bar{f}$ by a copositive Muntz polynomial.

If it is assumed that $f(0)=0$, or that the interval under consideration is $[a, b]$ with $a>0$, then it may be shown by proofs along the lines of those given 
above that it is not necessary to assume that any specific numbers are in $\Lambda$ :

COROLlary 1. If it is assumed in Theorems $1,2,3$ that $f(0)=0$, or that the interval under consideration is $[a, b], a>0$, then conclusions will follow for sets $\Lambda=\left\{\lambda_{0}, \lambda_{1}, \ldots\right\}$ of nonnegative real numbers with the following properties: (i) $\lim \lambda_{i}=\infty$; (ii) $\Sigma\left(1 / \lambda_{i}\right)=\infty$.

THEOREM 4. Let $f(x)$ be a continuous function on $[0,1]$ with the property that $\Delta^{j} f$ is alternately nonnegative and nonpositive on the intervals $\left(x_{0}, x_{1}\right)$, $\left(x_{1}, x_{2}\right), \ldots,\left(x_{k-1}, x_{k}\right)$ where $0=x_{0}<x_{1}<\cdots<x_{k}=1$. Let $\Lambda=$ $\left\{\lambda_{0}, \lambda_{1}, \ldots\right\}$ be a sequence of nonnegative real numbers with the following properties: (i) $0,1, \ldots, 2 k+j-1 \in \Lambda$; (ii) $\lim \lambda_{i}=\infty$, (iii) $\Sigma\left(1 / \lambda_{i}\right)=\infty$. Then given $\epsilon>0$ there is a corresponding Muntz polynomial $p(x)=\Sigma_{i=0}^{N} a_{i} x^{\lambda_{i}}$ such that $p^{(j)}(x)$ is alternately nonnegative and nonpositive on the same intervals as $\Delta^{j} f$, and such that $\|f-p\|<\epsilon$.

For $j=1$ this theorem reduces to Theorem 2. The theorem follows from Theorem 1, and the method of proof is an extension of that of Lemma 2. Here, $f^{*}$ is chosen to be a function in $C^{j}[0,1]$ with the property that $f^{(j)}$ is alternately nonnegative and nonpositive in the same intervals as $\Delta^{j} f$, and such that $\left\|f-f^{*}\right\|$ $<\epsilon / 2$. $q(x)$ is then chosen (by Theorem 1 ) to be a copositive Muntz approximation to $g(x)$ where

$$
g(x)=\frac{d^{j}}{d x^{j}} f^{*}(x), \text { and } q(x)=\sum_{i=0}^{N} b_{i} x^{\lambda_{i}},
$$

$\lambda_{i}^{\prime} \in \Lambda^{\prime}=\left\{\lambda_{i}-j ; \lambda_{i} \in \Lambda\right.$ and $\left.\lambda_{i} \geqslant j\right\}$ and $\|g-q\|<\epsilon / 2$. Let

$$
p_{1}(x)=\int_{0}^{x} q(x) d x+\frac{d^{j-1}}{d x^{j-1}} f^{*}(0) \text { and } p_{2}(x)=\int_{0}^{x} p_{1}(x) d x+\frac{d^{j-2}}{d x^{j-2}} f^{*}(0) \text {. }
$$

Iterate the procedure until we let

$$
p(x)=p_{j}(x)=\int_{0}^{x} p_{j-1}(x) d x+f^{*}(0) .
$$

Then

$$
\begin{aligned}
\left\|p-f^{*}\right\| & =\left\|\int_{0}^{x}\left[p_{j-1}(x)-\frac{d}{d x} f^{*}(x)\right] d x\right\| \leqslant\left\|p_{j-1}-\frac{d}{d x} f^{*}\right\| \\
& =\left\|\int_{0}^{x}\left[p_{j-2}(x)-\frac{d^{2}}{d x^{2}} f^{*}(x)\right] d x\right\| \leqslant\left\|p_{j-2}-\frac{d^{2}}{d x^{2}} f^{*}\right\| \\
& \leqslant \cdots \leqslant\left\|p_{1}-\frac{d^{j-1}}{d x^{j-1}} f^{*}\right\| \leqslant\|q-g\|<\frac{\epsilon}{2},
\end{aligned}
$$

and $p$ is the desired polynomial.

A quantitative theorem on monotone approximation is more simply stated 
with the help of some preliminary notation. Let $\Lambda=\left\{\lambda_{0}, \lambda_{1}, \ldots\right\}$. Let $P_{N, 0}=$ $P_{N, 0}(\Lambda)$ be the set of all Muntz polynomials $p(x)=\Sigma_{i=0}^{N} a_{i} x^{\lambda_{i}}$ satisfying $p(x) \geqslant 0$ on $[0,1]$; let $P_{N, j}=P_{N, j}(\Lambda), j=1,2, \ldots$, be the set of all Muntz polynomials $p(x)=\Sigma_{i=0}^{N} a_{i} x^{\lambda_{i}}$ satisfying $p^{(j)}(x) \geqslant 0$ on $[0,1]$. The degree of monotone approximation $E_{N, j}$ of a function $f \in C^{j}$ such that $\Delta^{j} f \geqslant 0$ is given by

$$
E_{N, j}(f, \Lambda)=\inf _{p \in P_{N, j}(\Lambda)}\|f-p\|, \quad j=0,1, \ldots .
$$

THEOREM 5. If $\Lambda=\left\{\lambda_{0}, \lambda_{1}, \ldots\right\}$ is a sequence of nonnegative real numbers with $0,1, \ldots, j \in \Lambda$, and $\Lambda^{\prime}=\left\{\lambda_{i}^{\prime}=\lambda_{i}-j, \lambda_{i} \in \Lambda\right.$ and $\left.\lambda_{i} \geqslant j\right\}$, then for any function $f \in C^{j}$ with $\Delta^{j} f \geqslant 0$,

$$
E_{N, j}(f, \Lambda) \leqslant E_{N-j, 0}\left(f^{(j)}, \Lambda^{\prime}\right) .
$$

Theorem 5 may be proved in the same manner that Theorem 4 is proved. Let $q \in P_{N-j, 0}\left(\Lambda^{\prime}\right)$ be a Muntz approximation to $f^{(j)}$. Let

$$
p_{1}(x)=\int_{0}^{x} q(x) d x+f^{(j-1)}(0) \text { and } p_{2}=\int_{0}^{x} p_{1}(x) d x+f^{(j-2)}(0) ;
$$

iterate until $p(x)=\int_{0}^{x} p_{j-1}(x) d x+f(0)$. Then $p \in P_{N, j}(\Lambda)$, and

$$
\|p-f\| \leqslant\left\|p_{j-1}-f^{\prime}\right\| \leqslant \cdots \leqslant\left\|p_{1}-f^{(j-1)}\right\| \leqslant\left\|q-f^{(j)}\right\|,
$$

and the theorem follows.

The results of Bak and Newman [1], and Leviatan [3] may now be used to give estimates on the degree of monotone approximation to a function $f \in C^{p}$, $p \geqslant j$. In particular, Bak and Newman have proved the following:

Let $\Lambda=\left\{\lambda_{0}=0, \lambda_{1}=1, \ldots, \lambda_{j}=j, \lambda_{j+1}, \lambda_{j+2}, \ldots\right\}$ be a sequence satisfying the growth condition $\lambda_{k} \geqslant s k$ for $k>j$ where $s>2$. If $f \in C^{j}[0,1]$, then there is a Muntz polynomial $p(x)=\Sigma_{i=0}^{N} a_{i} x^{\lambda_{i}}$ such that $\|f-p\|<$ $A_{j} \epsilon^{j} \omega\left(f^{(j)} ; \epsilon\right)$ where $A_{j}$ is a constant depending only on $j, \epsilon=\exp \left(-2 \sum_{k=1}^{n}\left(1 / \lambda_{k}\right)\right)$, and $\omega(f ; \delta)$ denotes the modulus of continuity:

$$
\omega(f ; \delta)=\sup _{|x-y|<\delta}|f(x)-f(y)| .
$$

We may use this result and Theorem 5 to obtain the following result (by a proof similar to that of Lemma 1):

Corollary 2. Let $\Lambda=\left\{\lambda_{0}=0, \lambda_{1}=1, \ldots, \lambda_{j-1}=j-1, \lambda_{j}\right.$, $\left.\lambda_{j+1}, \ldots\right\}$ be a sequence satisfying the growth condition $\lambda_{k} \geqslant$ sk for $k \geqslant 2 j$ where $s>2$. If $f \in C^{j}[0,1]$ and $f^{(j)} \geqslant 0$ on $[0,1]$, then

$$
E_{n, j}(f ; \Lambda) \leqslant A_{j} \epsilon^{j} \omega\left(f^{(j)} ; \epsilon\right)
$$

where $A_{j}$ is a constant depending only on $j$, and $\epsilon=\exp \left(-2 \Sigma_{k=1}^{n}\left(1 / \lambda_{k}\right)\right)$.

ADDED IN PROOF. D. Leviatan has pointed out to us that Theorems 1, 2, 3 may be improved as follows: 
COROllary 1'. Condition (i) in Theorems 1, 2, 3 may be replaced simply by $\lambda_{0}=0$.

Proof. Condition (i) is not needed in $\mathrm{T} 1_{1}$, while in Lemma 2 we can make the variable change $t=x^{\lambda_{1}}$ and proceed with the proof for the sequence $\mu_{i}=\lambda_{i} / \lambda_{1}$; the same alteration may be made in Lemma 4 .

\section{REFERENCES}

1. J. Bak and D. J. Newman, Müntz-Jackson theorems in $L^{p}[0,1]$ and $C[0,1]$, Amer. J. of Math. 94 (1972), 437-457. MR 46 \#9605.

2. W. J. Kammerer, Polynomial approximations to finitely oscillating functions, Math. Comp. 15 (1961), 115-119. MR 23 \#A1187.

3. D. Leviatan, On the Jackson-Müntz theorem, J. Approximation Theory 10 (1974), 1-5.

4. K. Lim, Note on monotone approximation, Bull. London Math. Soc. 3 (1971), 366-368. MR 45 \#2380.

5. G. G. Lorentz, Bernstein polynomials, Math. Expositions, no. 8, Univ. of Toronto Press, Toronto, 1953. MR 15, 217.

6. G. G. Lorentz and K. L. Zeller, Degree of approximation by monotone polynomials. I, J. Approximation Theory 1 (1968), 501-504. MR 39 \#699.

7. E. Passow and L. Raymon, Monotone and comonotone approximation, Proc. Amer. Math. Soc. 42 (1974), 390-394.

8. The degree of piecewise monotone interpolation, Proc. Amer. Math. Soc. 48 (1974), 409-412.

9. E. Passow, L. Raymon and J. A. Roulier, Comonotone polynomial approximation, J. Approximation Theory 11 (1974), 221-224.

10. J. A. Roulier, Monotone approximation of certain classes of functions, J. Approximation Theory 1 (1968), 319-324. MR 38 \#4875.

11. O. Shisha, Monotone approximation, Pacific J. Math. 15 (1965), 667-671. MR 32 \#2802.

12. W. Wolibner, Sur un polynôme d'interpolation, Colloq. Math. 2 (1951), 136-137. MR 13, 343.

13. S. W. Young, Piecewise monotone polynomial interpolation, Bull. Amer. Math. Soc. 73 (1967), 642-643. MR 35 \#3326.

DEPARTMENT OF MATHEMATICS, TEMPLE UNIVERSITY, PHILADELPHIA, PENNSYLVANIA 19122 (Current address of E. Passow and L. Raymon)

MATHEMATICS RESEARCH CENTER, UNITED STATES NAVAL RESEARCH LABORATORIES, WASHINGTON, D.C. 20375

Current address (O. Shisha): Department of Mathematics, University of Rhode Island, Kingston, Rhode Island 02881 